\title{
Sind Zellkern und Zellplasma selbständige Systeme?
}

\author{
Von
}

Prof. Dr. Schiicking

in Pyrmont.

Eingegangen am 7. Juni 1906.

In Nachstehendem möchte ich im Anschluß an ältere Beobachtungen einige neue Tatsachen mitteilen, welche dafür sprechen, daß dem Zellkern wie auch ganz allgemein allen dureh Teilung sich vermehrenden Zelieinschliissen neben dem Protoplasma eine Selbständigkeit und in gewissem Sinne eine selbständige phylogenetische Entwicklung einzurüumen ist. Ich beginne mit dem Bericht uber Untersuchungen, die ich in den Jahren 1902-1904 an der Zoologischen Station zu. Neapel unternahm und bei denen ich festzustellen suchte, ob auch bei der elementaren Grundform des Organismus, beim Li der schädigende Ľinfluß nachzuweisen sei, deu artfremdes Protoplasma im Tierkörper anfeinander auszuiiben ptlegt.

Ich brachte unbefmehtete Eier von Asterias glacialis, Strongylacentrotus lividus und Arbacia pusiulosa, und ywar in drei Versuchen jedesmal je zwei verschiedene Arten, 12-24 Stunden in sterilisiertem Seewasser zusammen. Das Resultat dieses einfachen Verfahrens war folgendes:

Bei den beiden Versuchsanordnungen, in denen ich Seeigeleier mit Scesterneiern zusammengebracht hatte, war jedesmal eine größere Anzahl von Scesterneiern aufgelïst, während bei den Kontrolleiern von derselben Art und Provenienz nur wenige Eier anfgelöst waren. Die Eier der beiden Seeigelarten hatten nicht zerstörend aufeinander eingewirkt. Fs hatten also die artfremden Eier, bei donen dio Cytoplasmamenge im Verhältnis zur Kelmmasse geringer war, deletïre Wirkung anf die erheblich größeren Eier ansgetibt, bei denen die Kermasse relutiv geringer als die Ilasmamasse war. In clas Seewasser waren von den Eiern aus schädigende Stoffe nicht eingetreten, wie ein entsprechender Versuch zeigte. Dieses Versuchsresultat legte den Schluß nahe, daß die Eier, wenn sie in ihren entsprechenden Arten nicht nïher verwandt sind, durch direkten Kontakt des Plasmas zerstörend aufeinander wirken, und daß dieser Effekt offensichtlich nicht von der Menge des Cytoplasmas abhängt, sonderu möglicherweise von dem Verhältnis zwischen Kernmasse und Plasma abhängig ist. Es entstand die Frage, ob das kernlose Protoplasma artfremder Eier zerstörend aufeinander zu wirken vermöge. Nach dem Vorgang der Gebridier IIERTwig zerschiittelte ich Eier von Asterias und den erwähnten beiden Seeigelarten und brachte die kernlosen Fragmente der Seeigeleier mit ebensolchen von Asterias zusammen. Einen nachteiligen Einflu $\beta$ der kernlosen Partikel aufeinander konnte ich nicht feststellen. Wohl aber wirkten kernhaltige Eier der Echiniden 
zerstörend auf die kernlosen Eipartikel von Asterias und ebenso umgekehrt die Kernhaltigen Eier von Asterias auf die kernlosen Partikel der Echinideneier. Ich schließe darans, daß der schïdigende Einfluß von artfremden Eiern aufeinander rou den Zellkernen und nicht vom Cytoplasma ausgeht.

Wir wissen bereits, daß kernloses Protoplasma sich zu erhalten und zu erühren vermag. Dies ist z. B. bei Fumaric hygrometrica der Fall, bei der kernlose Teilsticke 6 Wochen am Leben bleiben und auch Stärke aufzulösen imstande waren. Kernlose Teilstïcke von Spirogyra. Fäden sah KuEms (1) noch assimilieren und sowohl Stärke auflösen als auch neu bilden, wenn die Teilstiicke Chlorophyll enthielten. VERWOrN (2) sah bei Difflagia kemlose Teilstickchen noch nach ó Stunden lange Pseudopodien ansstrecken und auf mechanische, galvanische und chemische Reize reagieren. Derselbe sah kernlose Protistenpartikel vollständige Autonomie und Unabhängigkeit vom Kern bekunden. Die Verdauung der kernlosen Teilsticke elfubrt indes nach Hofer (3) und Verwoun sowohl der Zeit als der Intensitït nach eine erhebliche Abnahne, sie ist aber nicht aufgehoben. Fein zerhackte Fumaria hygrometrica vermag anch ans Teilstuickchen, in denen kein unverletzter Kern vorhanden ist, nene Moospflämzchenindividuen auszubilden (4).

Wie verhält sich umgekchrt der vom Cytoplasma isolierte Kern? Wir seheñ bei der Befruchtung, daß der Spcrmienkern, nachdem er die Geißel beim Eindringen in die Eizelle verloren und nur noch das Ceñtrosoma behalten hat, im fremden Protoplasma zunächst anschwillt and bei den weiteren bekamten Befruchtungsorgingen in seiner Lebenstiitigkeit nicht hinter dem Tikern zuritcksteht.

Wir wissen forner, daß in der Tier- und Pflanzenwelt den Sätzen somnis cellula e cellula und somnis nucleus e nucleo die Sütze »jede Plastide aus einer l'lastides und wjedes Chromosoma ans einem Chromosoma zur Seite stehen. Dio Folgerung; dah die genannten Zelleinschlïsse ebenso wie das Cytoplasma individuelle selbständige Systeme in Rahmen der Zelle darstellen, wird dnrch eine Anzahl triftiger Gründe gestuitzt.

Wie würde es sich unter dieser Voraussetzung nun mit dem entwicklungsgeschichtliclien Zusammenhang dieser Systeme verhalten? Insbesondere, befinden sich diese Systeme im Gleichgewicht oder beherrscht eines die andern? Es möge mir gestattet sein, meine von den bisherigen Anschaunngen abweichende Auffassung dieses biologischen Grundproblems hier in Kürze zu entwiskeh.

Zur cinisormaßen erschöpfenden Bcantwortng dieser Frage wïrde es gewib noch grober Fortschritte in unsern Kenntuissen iiber das physikalischchemische Verhalten del Zelle bediufen. Selbstverstuindlich würde es nicht angehen, heute außer dem Zellverband existierende niedere organische Gebilde, \%. B. die Spaltpilze, als nächste Vorstufen dieser Zelleinschliisse anzusehen. Ohne $/$ weifel stehen die Bakterien mit ihrem groben Gehalt an Nucleinkörpern und iluem Mangel an einem nachweisbaren Kern den Zellkernen noch min nïchsten. Der Hinweis, daß die Bakterien selbständige osmotische Systeme sind, wiirde hiergegen nicht ins Gewicht fallen, da anch die Zellkerne solche osmotischen Systeme darstellen.

Zunächst gibt uns die $\ddot{A} h n l i c h k e i t$, die das Verhalten vieler Spaltpilze gegeniiber dem Zellprotoplasma mit dem Verhalten des Zellkerns zum letzteren zeigt, wertvoile Fingerzeige. Wenn die Bakterienzooglöa bei den Leguminosenwurzeln ibren schleimigen Infektionsschlanch dureh die Zellwandungen hindurch- 
schiebt, so fuittert die Zelle die Bakterien mit Asparagin und Zucker, um darïr wieder den von den Bakterien aus der Luft assimilierten Stickstoff in der Gestalt von Schleim als Zahlung zu empfangen (5). Solange dies Verhältnis dauert, können wir von einer Symbiose zwischen dem normalen Zellinhalt und den Spaltpilzen sprechen. Nach einiger Zeit verwandelt sich jedoch diese Symbiose in einen ganz einseitigen Parasitismus. Die Pfanze zerstört die in ihren Wurzelknöllchen wohnenden Bakterien und saugt ihren Inhalt aus. Die *Symbiose* stellte also aur ein voribergehendes Gleichgewichtsstadium dar.

Die berihmte sSymbiose zwischen Algen und Pilzen bei den Flechten ist ein fast einseitiger Parasitismus, in dem die Algen reichlich organische Nährstoffe an die Pilze abgeben und dafür nur dic von den Pilzen vermittels Siureabsonderung aufgelösten anorganischen Stoffe erhalten. Noch merkwürdiger ist das Zusammenleben von den niederen pflanzlichen Zellen der Algen und tierischen Zellen, wie wir sie bei vielen Seetieren beobachten $(6)$ Daß ein einseitiger Parasitismus den bewirtenden Organismus dazu führen kann, fuir die Pflege seines Gastes ohne Entgelt sehr komplizierte Eimichtungen zu treffen, schen wir an dem Beispiel der Insektengallen und Pilzgallen. Schließlich gehören zu den Vereinigungen, in denen die Wirtszelle den Parasiten sozusagen in Pflege nimmt, auch die syphilitischen und tuberkulösen Geschwiilste.

Die in der Einleitung mitgeteilten Beobachtungen lassen nun erkennen, daß bei den betreffenden Metazoen die Verdanungstätigkeit und gewisse Schutzeiarichtungen mit dem Zellkem in Verbindung stehen, von dem wir bisher sehon annabmen, daß el das formative aufbauende und fitr die Weiterentwicklung der Zelle und die Bildung der Zellhant bestimmende Agens ist.

Ist nun in der lebenden Zelle unter der Voransetzung, da es sich um selbstiindige Systeme handelt, ein Zustand gegeben, der eineu der beiden Komponenten einen mehr oder minder parasituren Charakter zuschreiben läßt, oder besteht ein dauerndes Gleichgewicht in der gegenseitigen Inansprnchnahne bei Kern und Plasma? Für diese Frage ist das Studium der Conjugation bei den Protozoen und der Befruchtung bei den Metazoen von besonderer Bedeutung:

Bekanntlich tritt die Conjugation der Protozoen, die ihr Analogon in der geschlechtlichen Vermehrung der Metazoen hat, nach voriibergehenden Hungerperioden auf, die eine gewisse Reifung des Kerns hervorufen (7). Überfitternng und andauernder Hnngerzustand verhindern diese Reifung des Kerns und haben eine übermäßige Vergrößerung desselben zur Folge. Die Organisation des Kerns gestattet nicht, daß dieser ohne Schädigung fiir das l'rotozoon danernd ein gewisses Größenmaß iiberschreitet. Bei einer solchen Vergrößerung des Kerns gehen die Protozoen, nachdem noch eine Zeitlang Zellteilungen in der betreffenden Kultur; aber keine Conjugationen unehr, zinstande gekommen sind, zugrunde. Wenn wir bei andauerndem Hungerzustand den Kern auf Kosten des in seinem Stoffivechselgleichgewicht gestörten nud daher geschwähten Plasmas sich vergrößern sehen, so denten diese Zustände, in denen der Kern dauernd vom. Plasma mehr exhält, als er demselben zurückgibt, auf frühere parasitäre Entwicklungsvorgänge, bei denen Kern und Plasma noch getrennte biologische Systeme waren. Anderseits exinnern gewisse Vorginge, die wir bei der Zellvermebrung im lebenden Gewebe bei uns beobachten - Erkrankungen der Gewebe bei danernder ibermäßiger Nahrungঞzufuhr, wie auch bei Inanitionszustinden -.. an die unter ähnlichen Bedingungen eintretenden Störungen des Verhiiltnisses zwischen Kern und Plasma bei den Protozoen und an die Folgezustinde dieser 
Störungen. Bei der den Pathologen bekannten sog. Wucheratrophie beobachten wir bei Hungerzuständen und fieberhaften Krankheiten schon nach mehreren Tagen hochgradige Wucherung der Zellkerne, so im Fettgewebe, ebenso in den Mnskelfasern, bei denen in den Primitivbiindeln im unveränderten Sarcolemmschlanch Protoplasmamassen mit stark gewucherten Kernen entstehen. Ähnlich verhalten sich die Kerne dex Schwansschen Scheide in den Nervenfasern. Dieselben Kernwucherungen beobachten wir an andern Stellen im Inanitionszustand, z. B. sehr deutlich bei der Atrophie der Geschmacksknospen.

Durch Aushungerung und Schwrichung des Protoplasmas werden also allgemein die Kerne zur Vergrößerung und Teilung angeregt, ehe das Gewebe zagrunde geht. Der Umstand, daß dieser Prozeß sich vielleicht als niitzlich fiir den Gesamtorganismus erweisen kann, stellt die Selbständigkeit des Kerns und seinen parasitiiren Charakter nicht in Frage, ebensowenig wie z. B. das für das Gesamtgebilde nützliche Resultat der Flechtenbildung als Beweis gegen das selbständige Verhalten der Flechtpilze herangezogen werden kann.

Das im natiurlichen Ablanf der Entwicklung erfolgende $A b$ sterben der Gewebe und des gesamten Somas der Metazoen orscheint unter diesem Gesichtspnnkt als eine Folge der bei den mannigfachen Störugen des Stoffwechsels nicht wieder rückgängig zu machenden Anfhebnng des Gleichgewichts zwischen Kera und Plasma. Der Tod wäe somit das SehluBresultat der während der Lebensdaner eintretenden Stürungen des Kemplasmagleichgewichts. Besonderes Interesse mïssen bei dieser Anschanung nuzweifelhaft die bei der Geschwulatbildnng beobachteten Gewebsverïaderungen erregen, die sich als eiu extremer Fall des schließlich stets eintretenden Mißverhältnisses zwischen Kern und Plasma charakterisicren. Das unbekannte geschwulsterzengende „Incitament nenerer Antoren, das vielleicht in gewissen leicht assimilierbaren oder die Assimilationsf:ihigkeit der Kerne steigernden Nncleinkürpern besteht, wiirde nicht etwa das Wachstum der Kerne ron prïformierten Carcinom-oder Sarcomzellen fördern, sondern nach meiner Anschanung imstande sein, jedem Kern gewisser normaler Zellarten ein zur Geschwulstbildung fiihrendes Wachstum zu verleihen. Die gesteigerte Virnlenz des Mïnsecarcinoms nach wiederholten lassagen wïde sich durch die infolge der giinstigen sperifischen Erährungsbedingungen gesteigerte Selbstindigkeit der Kerne der transplantierten bzw. geimpften lebonden Krobszellen erklären. Die Immunität mit Kiebszellen geimpter Miuse und Ratten endlich wïre als das liesultat der im Ilasma vor sich gehenden, zur lbehauptung des Gleichgewichts gegen die Kerwergrößerung gerichteten Stoffwechselvorginge zu deuten.

Nach dieser Auffassung würde also der Zellkern selbst die Rolle des gesuchten Geschwulstparasiten spielen.

Die Spezifaituit der Zellbestandteile, wie sie bekanntlich jeder Art eigentïmlich ist, kann hier nicht als Gegengrund herangezogen werden, denn eine solche beobachten wir auch bei ausgesprochen parasitiren Vereinigungen, boi denen nur ganz bestimmte Organismen der einen Art mit bestimmten Organismen der andern Art sich zusammenschließen.

Bei der Befruchtung der Metazoen verlaufen im großen und ganzen die Vorginge nicht anders wie bei der Conjugation der Protozoen; nur diejenigen Kerne schließen sich einander an, die durch Verkleinerung auf die Hälfte bzw. anreh Reduction der Chromosomenzahl dio erforderlichen Vorbereitungen darn getroffen haben. Wir sehen, daß bei den Protozoen diese Reifung und Teilung 
der Kerne durch übermäßige Ernährung und Aushungerung verhindert werden kann. Vielleicht spielen auch noch andre ungünstige äußere Einwirkungen dabei eine Rolle. Bei den Metazoen ist indes die Einrichtung entstanden, daß gewisse, schon in den ersten Entwicklungsstadien besonders giinstig gelagerte Zellen, die späteren Keimzellen, von den Wechselfällen änßerer Einflüsse nnabhängig werden und daher unter allen Umständen ihre Kerne reifen und teilen lassen können. Bei der zweigeschlechtlichen Befruchtung der Metazoen lösen sich- die in ihrer Kernmasse bzw. ihren Chromosomen nm die Halfte reduzierten Keimzellen vor ihrer Vereinigung von ihrem Sitz ab. Die Geschlechtsdifferenz erscheint als eine Folge des Ablösungsprozesses und der Notwendigkeit, durch möglichst günstige mechanische Verhältnisse die Vereinigung der Chromosomen zu. sichern. Zur Erreichung dieses Resultats treten sekundiir die mannigfachsten organischen und sonstigen Einrichtungen, wie sie z. B. bei der Befruchtung der Seeigeleier von mir festgestellt wurden, ein. Diese Differenzierung dor Beweglichkeit, d. h. die Schaffung eines unbeweglichen Eies gegenüber einer möglichst beweglichen Spermie, war nur durch Änderung des Verhältnisses zwischen Plasmamasse und Kernmasse zu erreichen. Die hieraus gezogene Folgerung; daß sich nur mechanische und quantitative Unterschiede in letztem Grunde in den Geschlechtsverschiedenheiten ausdriicken, will indes nichts besagen, so lange die These nicht widerlegt ist, daß es einen Punkt gibt, an dem quantitative Unterschiede zu qualitativen werden. Durch Zunahme des Plasmas der weiblichen Keimzelie und Zunahme der Kernmasse der männlichen Keimzelle scheint nach den bisherigen Beobachtungen die Entwicklnng des weiblichen Geschlechts, durch die umgekchrten Verhältnisse, Abnahme des Plasmas der weiblichen Keimzelle und Abnahme der Kernmasse der münnlichen Keimzelle die Entwicklung des münnlichen Geschleehts begiinstigt zu werden.

Nenere Versuche von Godiewsri jun. Iehren, daß bei Anwesenheit väterlicher Chromosomen und bei fehlendem Eikern, muitterliche Charaktere vererbt werden können. Ganz meinen Ausfuhrungen entsprechend verbieten diese Versuche, dem Kern eine ausschließliche Rolle bei der Vererbung zuzuschreiben, lassen vielmehr erkennen, daß anch das Plasma für die Vererbung von Bedentung ist und geben damit eine wichtige Unterstïtzung fuir meine Auffassung, daß Kern und Plasma individnelle, wonn anch anfeinander angewiesene systeme bilden.

Eine weitere Stiitre dieser Auffassung ist die ron mir festgestelite Tatsache, daß die Vereinigung ron ki nnd Sperma bei den Echiniden durch die Verschmelzung von beiderscitigen Plasmateilen eingeleitet wird (8).

Anf die Frage, warum die Kerne bei Protozoen und Metazoen in Verbindung miteinander treten, gibt uns die zunehmende Hiiufigkeit der Verschmelzung der entstehenden Individuen in der anfsteigenden Organismenreiho Antwort. Auf den niedersten Stufen des organischen Lebens, bei den kernIosen, aus Nucleinen zusammengesetzten Bakterien und verwandten Formen, sehen wir jede Vereinigung der durch Teilung entstandenen Individuen ausbleiben. Erst bei den J'rotozoen mit jhrer Zweiteilng in Kern und Plasma tritt Conjugation ein und zwar, den noch wenig entwickelten Kern- und Plasmasystemen entsprechend, nur unter gewissen Bodingungen und in gewissen Zeiträumen. Bei den Metazoen endich mit ihren höchst differenzierten Kern- und Plasmasystemen tritt Entwicklung ohne Befruchtung mit wenigen Ausnahmen iiberhaupt nicht mehr ein. Der festgefiigte molekulare Apparat der keinem I'lasmasystem angeschlossenen Kernsubstanz der Bakterien erlanbt anscheinend eine unbegrenzte 
Vermehrung derselben ohne zeitweilige Korrekturen. Mit der Vereinigung von Kern und Plasma und dem Ausbau des Kerns in ein Bündel individueller Chromosomen aber tritt die Möglichkeit einer übermäßigen Differenzierung in dem schwankenden Verhältnis zwischen den beiden selbständigen Faktoren ein. Diese zerstrenende und artauflösende Wirknng der wechselnden Beziehungen zwischen den beiden wichtigsten Bestandteilen der Zelle macht eine bestiandige arterhaltende Korrektur notwendig, wenn die Art die zu ihrer Erhaltung notwendige Konstanz der Kernplasmabeziehungen sich bewahren will. Diese sich immer wiederholende Korrektur und Ansgleichung der eingetretenen Differenzierungen ist die Befruchtung. Es ist also das Gegenteil von dem zutreffend, was die bisherigen Befruchtungstheorien behaupten. Nicht die durch $\gg$ Amphimixis "geforderte Differenzierug ist das Resultat der Befruchtung, sondern die Bekamp fung der Differenzierung and die Wiederherstellung des gostörten Gleichgewichts zwischen Kern und Plasma.

Die Befruchtung kann die Differenzierung im Rahmen der Art fördern, aber dariber hinans wirkt sie als Regulator, nicht artvermehrend, wie man bisher annahm, sondern artbeschränkend and artixicrend. Die Fille, in denen die Befruchtung die Entwicklung newer Arten zu merstitzen scheint, sind seltene Auswahmen gegeniiber der ungeheueren Mehrzahl der Fille, in denen das Gegenteil stattfindet. Schon verhiilnismäßig geringe Abweichungen, die zwei Individuen in ihrem Ban voneinander zeigen, geniugen bekanntlich, un ihre geschlechtliche Vereinignug zu einer unfuchtbaren zu machen oder sie sterile Bastarde erzengen zu lassen. Nach der Lehre von der Amphimixis miißten aber die Bastarde vorzugsweise den supponierten Befuchtungszweck, der Differenzierung, zu dienen imstande sein. Da die Korrektur des gestörten Kornplasmaverhälnisses nur bei den Keimzellen eintritt, so bleiben nur diese ewig. jung und in gewissem Sinne unsterblich, während die Gesamtheit der nicht durch die Befruchtung regenerierten Zellen drurch immer weitergehende Gleichgewichtsstörungen zwischen ihren Komponenton zugrunde geht.

\section{Literatur.}

1) Kruns, Über den Einflaß des Kerns in der Zelle. Biol. Centralbl. Bd. VII. 1887.

2) Verworn, Die physiologische Bedentung des Zellkexus. Archiv f. d. ges. Physiologic. Bd. LI. 1891.

3) Hower, Experimentelle Untelsuchungen iber den Einfuß des Kerns anf das Protoplasma. Jenaische Zeitsehr. f. Naturwissenschaft. Bd. XXIV.

4) Nussbaum, Über die Teilbarkeit der lebenden Materie. Archiv f. mikr. Anat. Bd. XXVI. 1886.

5) B. Fraxk, Über die Pilzsymbiose der Leguminosen. Landwirtschaftl. Jahnbiicher. 1890.

6) Brandr, i̛ber das Zusammenleben von Tieren und Algen. Verh. d. plysiol. Gesellsch. zu Berlin. 1881.

7) Ricmard Horwwig, Über die Conjugation der Infusorien. Abhandl. d. bayer: Akad. d. Wiss. II. Kl. Bd. XVII. 1889.

8) Sutücking, Zux Physiologie der Befruehtung, Parthenogenese und kntwicklung. Archiv f. d. ges. Physiologie. Bd. 97. 